\title{
"Ya nO hay ÁRbOles ni AgUA". Perspectivas de los CAMBios AMBIENTALES EN COMUNIDADES DE ZiNACANTÁN, CHIAPAS
}

\author{
Gerda Ursula Seidl, Helda Morales, Luis Alfredo Arriola Vega, \\ Angélica Aremy Evangelista García
}

Resumen: El artículo se basa en un trabajo empírico en donde se indaga sobre perspectivas de los cambios ambientales entre hombres y mujeres, ancianos, adultos y jóvenes en cuatro localidades del municipio de Zinacantán, en Los AltosdeChiapas.Parael trabajodecamposeemplearon métodos cualitativos, sobre todo entrevistas semiestructuradas y talleres. Los entrevistados identificaron como problemas principales el cambio productivo, la degradación y disminución de bosques y agua, el cambio en la alimentación, problemas con el clima y con la contaminación por desechos. Hay diferencias entre los parajes, debido a su situación económica y ambiental desigual. Existen además discrepancias entre los géneros y generacionesen la identificación de los cambios ambientales. Las diferencias encontradas indican que no hay homogeneidad dentro de un mismo grupo cultural y que es importante distinguir entre géneros, generaciones y demás variables, sise quiere llegar aun diseño adecuado de programas y proyectos.

Palabras clave: Perspectivas ambientales, percepciones ambientales, género y medio ambiente, cambios ambientales.

Enviado a dictamen: 04 de abril de 2011

Aprobación: 25 de mayo de 2011

Revisiones: 2

Gerda Ursula Seidl. Maestra en Ciencias en Recursos Naturales y Desarrollo Rural en El Colegio de la Frontera Sur, México, línea de investigación: Género y Medio Ambiente, Sociedad y Ambiente. Correo electrónico: gerdiseidl@yahoo.com.

Helda Morales. Doctoraen Manejode Recursos Naturales, líneadeinvestigación:Agroecología, Manejo sustentable de los recursos naturales. Correo electrónico: hmorales@ecosur.mx. Luis Alfredo Arriola Vega. Doctoren Antropología por El Colegiodela FronteraSur,Sede Campeche, línea deinvestigación:MigracionesenlafronterasurdeMéxico.Correoelectrónico:larriola@ecosurmx. Angélica Aremy Evangelista García. Doctoraen Ciencias Socialesy Humanidadesporel Centro de Estudios Superiores de México y Centroamérica (CESMECA), línea de investigación: Género,Jóvenes, Salud sexual y reproductiva. Correo electrónico: aevangel@ecosur.mx.
Abstract: This article is based on empirical work comparing perspectives on environmental changes between men and women, elderly, adult and young people in 4 communities in the highlands of Chiapas. Qualitative methods were used for fieldwork, especially semi-structured interviews and workshops. The participants identified as the most urgent problems declining productivity, the degradation of forests and water, nutrition, climate change and garbage. Differences among the communities stem from their unequal economic and environmental situation. There were also gender differences and differences among generations. The results indicate that there is no homogeneity within cultural groups, and that it is important to consider differences among genders, generations and other groupings when designing programs and projects.

Keywords: Environmental perspectives, environmental perceptions, gender and environment, environmental changes.

\section{Introducción}

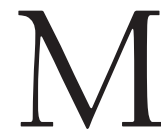
uchas veces, las políticas ambientales o intervenciones de organismos gubernamentales y no gubernamentales quedan sin tomar en cuenta la visión de las personas sobre su ambiente y los cambios que están ocurriendo en él. En consecuencia, proyectos diseñados con las mejores intenciones fracasan o son de poco o ningún beneficio para las personas. Por lo tanto, para una toma 
de decisiones adecuada es de suma importancia conocer las valoraciones y opiniones de la gente local.

Existen varios estudios en México sobre la visión que las personas tienen del ambiente, generalmente con el concepto de percepciones ambientales (por ejemplo, Arizpe, 1993; Benez 2008; Chávez Ballado, 2007). Dichos estudios encuentran una gama amplia de puntos de vista, sentimientos y opiniones en la población, que van "de extremo a extremo" (Lazos y Paré, 2005: 108). Durand (2008: 82) sugiere acercarse al fenómeno de la visión y vivencia del ambiente con el concepto perspectivas ambientales, ya que éste puede dar cuenta de la diversidad de visiones existentes en grupos supuestamente homogéneos, y vincular el nivel de la experiencia directa - percepción - con el de la interpretación. Es dicho nexo el que imprime/otorga su característica distintiva a este paradigma. Por ello, desde este enfoque el presente trabajo se propone indagar cómo personas de cuatro comunidades del municipio de Zinacantán, en Los Altos de Chiapas, perciben e interpretan los cambios ambientales para conocer cuáles son cambios prioritarios y problemáticos para ellos.

Los estudios sobre percepciones ambientales comúnmente no hacen énfasis en la cuestión de los cambios de este tipo. Sin embargo, en todos los estudios que utilizan esta perspectiva el cambio ambiental es algo subyacente, ya que el estado actual, sobre el cual los sujetos se expresan, siempre es resultado de cambios continuos. De hecho, el cambio es algo normal e inherente a cada ambiente, ya que si asumimos que el ambiente es producto de actividades o eventos pasados tenemos que comparar el ambiente no tanto como un contenedor de objetos sino como una escultura en obra continua, nunca terminada y trabajada por muchas manos (le debemos esta metáfora a Ingold, 1997: 50).

\section{El lugar de estudio}

Este estudio se llevó a cabo en el municipio de Zinacantán, en Los Altos de Chiapas (ver Figura 1). Es uno de los municipios chiapanecos con población casi exclusivamente indígena, mayoritariamente tsotsiles, en donde existe un desarrollo relativamente fuerte pero desigual en términos económicos y de infraestructura, debido a una conversión productiva en los últimos treinta años, aproximadamente. Esta conversión, descrita por Collier (1992) y Collier, Mountjoy y Nigh (1994), consiste sobre todo en el cambio de cultivo de milpa - para subsistencia y para venta - al trabajo remunerado en construcción y servicios. Además en los parajes grandes y en varios pequeños de la parte noreste del municipio cobró importancia el cultivo de flores bajo invernadero, que se volvió la primera fuente de ingresos en estas localidades desde la década de 1990. Aunque la superficie destinada a floricultura es relativamente pequeña, supera los ingresos generados por los demás productos: en 2002, 53.41\% del valor de la producción agrícola provenía de las flores, mientras que el maíz — 93.02\% de la superficie sembrada - solo aportaba 32.94\% (Anuario Estadístico de Chiapas, 2002).

Debido, en parte, a este cambio productivo, a la poca planeación y a la falta de servicios, hay una escasez y contaminación notable de los recursos naturales, como suelos y agua, acompañado de una deforestación acelerada (Burguete, 2000). Esto ha llevado a conflictos fuertes entre diferentes grupos - ya sea por su afiliación política o por su adscripción religiosa- debido al recurso agua que escasea cada vez más, y por otros recursos como bosques, terrenos e incluso por ciertos servicios, como la recolección de basura (Burguete, 2000; Rincón García, 2007). En las últimas décadas ocurrió un notable crecimiento poblacional que influye en las transformaciones del ambiente. Hacia 1920 vivían 1,500 personas en el municipio, cifra que ascendió a 13,006 personas para 1980; el último censo de 2010 registró 36,489 personas en Zinacantán (INEGI, 2010).

Zinacantán se caracteriza por una relativa heterogeneidad de actividades económicas que llevan a cabo sus pobladores y por sus condiciones ambientales. Hay una gran diferencia en estilos de vida, de consumo 
y fuentes de ingreso, entre los poblados semiurbanos y las comunidades más pequeñas. También existen diferencias en los poblados en lo que respecta a la disponibilidad y calidad de agua, el tipo de viviendas, la disponibilidad de leña u otro combustible y la contaminación por basura, aspectos que influyen en la construcción de distintas perspectivas ambientales y en los problemas que enfrentan las comunidades.

En el Cuadro 1 se presentan las principales diferencias y similitudes entre los lugares en donde se hizo este estudio. Zinacantán cabecera tiene 3,876 habitantes y Nachig 3,260; mientras que en Jech ch'entik y Elambo Bajo viven 892 y 629 personas, respectivamente. Las comunidades semiurbanas ${ }^{1}$ estudiadas cuentan con servicios de agua entubada, drenaje y de recolección de basura, mientras que las comunidades rurales no tienen acceso a ello. También la actividad económica principal varía: mientras que en la cabecera y en parajes aledaños predominan la floricultura y el trabajo en servicios, en algunas de las comunidades más rurales hay una mezcla entre floricultura, cultivo de milpa, hortaliza y frutales, y de trabajo foráneo en construcción y servicios, aunque en menor grado. En todas las comunidades se elaboran artesanías textiles, trabajo exclusivo de mujeres y niñas.

La cosmovisión tradicional católica aún sigue fuertemente enraizada en la mayoría de los zinacantecos. Pero, por cambios culturales de índole religioso o generacional, esta cosmovisión se va transformando más y más. Las diferentes visiones que tienen las personas a menudo resultan en conflictos sobre los recursos naturales protagonizados en la esfera pública principalmente por personas de distintas filiaciones religiosas o políticas. Por estar inmersos en un mundo diferente al de sus antepasados, y por la educación formal que desvaloriza dicha cosmovisión, este desdibujamiento de la cosmovisión tradicional zinacanteca está ocurriendo también entre los jóvenes.

\section{La lente teórica: perspectivas ambientales}

Si lo que interesa es la forma de entender la naturaleza, Leticia Durand (2008) sugiere abandonar el término de percepciones ambientales y sustituirlo por el de perspectivas ambientales, que incluye percepción e interpretación. Según esta propuesta, los humanos no habitamos un mundo culturalmente construido en su totalidad, sino también una sociedad y una cultura naturalmente construida, o sea que naturaleza y cultura se influyen y "construyen" mutuamente.

La autora sugiere el concepto de perspectivas ambientales, que define como "el conjunto de normas, supuestos y valores que resultan de la vivencia del entorno natural y permiten comprenderlo y explicarlo" (Durand, 2008: 82). Las acciones y vivencias individuales forman parte de la experiencia de los individuos y "son de suma importancia en la conformación de las percepciones sobre la naturaleza" (Durand, 2008: 82). La vivencia con el entorno natural corresponde a la percepción, mientras que el conjunto de normas, supuestos y valores, así como la comprensión y explicación, corresponden a la interpretación. Sin embargo, es muy difícil separar empíricamente los dos componentes, ya que la percepción y la interpretación de lo percibido están íntimamente relacionadas.

Tanto para Leticia Durand como para Tim Ingold percepción es acción. Los autores se refieren aquí al concepto de affordances - oportunidades ambientalesque viene originalmente de la psicología ecológica -J. Gibson, en los años 60-, y asevera que "lo que percibimos está en función directa a la forma en la que actuamos, y percibimos aquello que los objetos nos ofrecen en el contexto de la acción en la que nos encontramos" (Durand cita a Ingold, 2008: 80). Este aspecto se vuelve importante cuando se tratan de explicar las diferencias encontradas en un mismo grupo cultural. iPor qué las personas de Elambo tienen otras perspectivas sobre el ambiente que las personas de Zinacantán cabecera, por ejemplo? O ipor qué las 
mujeres enfatizan problemas ambientales distintos a los de los hombres? Más adelante veremos que la explicación está estrechamente relacionada con el hecho de que las interacciones con el ambiente son diferenciadas $y$, por ende, las perspectivas resultan desiguales.

\section{Hacia una definición de ambiente}

En el presente estudio nos enfocamos en el ambiente biofísico - natural y construido - de las personas en Zinacantán. Para el ambiente biofísico, retomamos la definición de ambiente que usa Pamela Hartigan: "El ambiente biofísico incluye el 'espacio vital' natural y el construido - o 'edificado'- donde los hombres y las mujeres llevan a cabo sus actividades. Incluye elementos naturales y edificados, como árboles, fuentes de agua, mosquitos, casas, chimeneas, fábricas y calles (...)" (Hartigan, 1998: 7).

Tim Ingold resalta cómo un aspecto importante en el ambiente es la encarnación de actividades pasadas: "La historia de un ambiente es la historia de todas las actividades de todos los organismos, humanos o no, contemporáneos o ancestrales, que han contribuido a su formación" (Ingold, 1997: 50). Esta definición de ambiente encuentra su eco en la concepción de los zinacantecos cuando hablan de su ambiente como "la tierra de nuestros ancestros" -balamil jtotik jme'tik-, tierra que tiene historia, de la cual además se derivan ciertas normas para su manejo y manipulación futura.

El concepto ambiente no tiene un referente directo en la cosmovisión tsotsil, y carece de traducción. Según nuestros informantes, "ambiente" sería la traducción de algo como "balamil" - la tierra-, "ch'iel kuxlejaltik" —nuestra vida que hay-, o "bolometik jabnaletik" los animales y árboles-. Inclusive el concepto de naturaleza tampoco tiene equivalente directo en tsotsil. En el trabajo de campo se usó el concepto ambiente en este sentido amplio, y se tradujo, dependiendo del contexto o el enunciado particular en el que fue utilizado, como "tierra", "vida", "animales y árboles".

\section{Cambios ambientales}

Para los propósitos de esta investigación se definen como cambios ambientales todos los procesos de transformaciones, deterioros o mejoras, que han sido identificados por los sujetos en su ambiente natural y construido; se refiere sobre todo a la dimensión vital, directa, inmediata de los sujetos, a los espacios que ellos y ellas cotidianamente usan, construyen, conocen y significan, pero también a transformaciones que identificaron y expresaron en el ambiente humano.

Para poder recabar la visión tanto de ancianos como de jóvenes, no se restringió la escala temporal a propósito, sino se dejó que ésta dependiera de cada sujeto. Si preguntamos a una persona anciana de 70 años, por ejemplo, sobre cómo ha cambiado su comunidad desde que era niña o niño, el periodo probable para el cual relata cambios es de 60 a 65 años, mientras que una persona joven de 20 años probablemente platica de cambios ocurridos en los últimos 15 años.

\section{Género y medio ambiente}

Para indagar las opiniones y valoraciones de hombres y mujeres frente al ambiente, utilizamos una perspectiva de género, ya que hombres y mujeres muchas veces "tienen intereses completamente distintos en el cambio ambiental, en general, y en las políticas específicas de intervención del manejo de recursos naturales, en particular" (Joekes, 2004: 496). En este sentido, el enfoque de género permite "visibilizar lo que normalmente no es visible" (Tuñón, 2000), o sea los intereses, visiones y estrategias posiblemente diferenciadas entre hombres y mujeres. Varios estudios empíricos, desde diferentes enfoques, señalan una estrecha vinculación entre los factores género y medio ambiente (Martínez, 2000; 
Vázquez, 2003; Joekes, 2004). Se pueden identificar tres corrientes teórico conceptuales que tratan de entender la relación de las mujeres y los hombres con la naturaleza. Estas tres corrientes son: el Ecofeminismo, Mujeres y Medio Ambiente, y Género y Medio Ambiente (Rivera, 1998; Vázquez y Velásquez, 2004). Aunque no nos adscribimos de manera pura a ninguna de estas corrientes, utilizamos partes de ellas porque nos permiten explicar las diferencias de perspectivas existentes entre hombres y mujeres.

\section{Metodología de la investigación}

Para el trabajo de campo se usaron herramientas etnográficas como la observación participante, pláticas informales, entrevistas semiestructuradas y talleres con grupos de mujeres y hombres zinacantecos de diferentes edades. El punto de llegada fue el grupo de señoras Mujeres Sembrando la Vida, que se organizaba alrededor del microcrédito y con las cuales una de las autoras tenía relaciones laborales previas. De allí se fueron extendiendo los contactos hacia sus hijos, hijas, esposos, y hacia un grupo de estudiantes de preparatoria.

Fueron entrevistadas 16 personas: 9 mujeres y 7 hombres. Después de las entrevistas se realizaron 9 talleres con un total de 117 personas participantes -103 mujeres y 14 hombres-. Somos conscientes de que el número desigual entre mujeres y hombres puede provocar un sesgo en los resultados. En los Cuadros 2 y 3 se especifica el número de participantes, la localidad de donde provienen y su edad.

En las entrevistas se indagó sobre los cambios ambientales prioritarios paralos pobladores. Explorando las dimensiones temporales "antes-ahora-después", se les pidió a los informantes un relato histórico enfocado en su ambiente biofísico. A partir de un primer análisis de las entrevistas, se identificaron cuatro temas clave que fueron mencionados repetidamente o con especial énfasis.
1. La problemática productiva, especialmente el cambio de milpa a floricultura, y la pérdida de productividad de la milpa.

2. La escasez y contaminación del agua.

3. La deforestación o empobrecimiento de los bosques.

4. La contaminación por desechos sólidos — basura.

Muchas de las personas que participaron en el estudio no sabían leer ni escribir y eran monolingües, especialmente las mujeres adultas, y hombres y mujeres ancianas. Todas y todos son hablantes de tsotsil. En el trabajo con personas analfabetas o personas que están poco acostumbradas a textos escritos ha resultado muy provechoso trabajar en grupos y usar "códigos" visuales como provocadores de participación, diálogo, análisis y autodiagnóstico. Por eso usamos en los talleres una metodología que viene de la Educación Transformadora, una vertiente de la Educación Popular que fue desarrollada en África por Anne Hope, Sally Timmel, y otros integrantes del Training for Transformation Institute (Hope y Timmel, 1995).

Para los cuatro temas mencionados arriba se elaboraron pares de fotografías que representaban los cambios ambientales, mismos que fueron utilizados como códigos visuales en los talleres. Por ejemplo, para la deforestación se buscó una fotografía con un bosque de pino encino típico de Los Altos, y se colocó junto a una fotografía con un paisaje deforestado. Después de mostrar las imágenes inició una discusión abierta, no obstante guiada, entre los y las participantes. Esta metodología resultó muy llamativa y propició la colaboración vivida de las personas que participaron en el estudio.

Los talleres, así como las entrevistas, fueron audiograbados, transcritos y, en el caso de las entrevistas y talleres en tsotsil, traducidos. Se llevaron a cabo cinco talleres y seis entrevistas en tsotsil. Después de la transcripción se procedió al análisis de los datos, identificando categorías de análisis y comparando los datos encontrados entre hombres, mujeres, jóvenes, 
adultos, ancianos, y entre las personas de diferentes comunidades.

\section{Hallazgos: Los principales cambios ambientales}

Además de la problemática productiva, la escasez y contaminación del agua, la deforestación y la basura, la mayoría de los participantes señalaron repetidamente problemas relacionados con cambios meteorológicos; por ejemplo, menos lluvia y temperaturas más extremas, así como cambios en la alimentación, enfermedades, y los cambios en la imagen urbana, que se refiere a calles, caminos y casas. En el Cuadro 4 se muestra de manera resumida lo que los participantes mencionaron como cambios ambientales, y sí fueron identificados mayoritariamente como un problema o conflicto o, por el contrario, como una ventaja o un cambio positivo. También se señalaron algunas causas y efectos que ocasionaron los cambios.

Debido a limitaciones de espacio, el presente artículo se ciñe a los hallazgos concernientes a cambios en las estrategias productivas, así como al agua, los bosques, y la contaminación por desechos - basura-. Finalmente, procederemos a describir las diferencias encontradas entre parajes, géneros y generaciones.

\section{Cambios en las estrategias productivas}

El cultivo de milpa en Zinacantán disminuyó considerablemente debido a una serie de transformaciones económicas (Collier, 1992) a partir de mediados de la década de 1970. Primero, el cultivo de maíz disminuyó en las tierras bajas e incrementó en las tierras altas. ${ }^{1}$ Segundo, se generalizó el empleo de agroquímicos y se disminuyó la mano de obra implicada. Las maneras de cómo trabajar la milpa han cambiado radicalmente, y en general el cultivo del maíz ya no tiene la importancia económica central que solía tener. Sin embargo, en las comunidades donde no hay alternativas de empleo — trabajo en servicios o floricultura - la producción de maíz sigue siendo muy importante, y allí los entrevistados y participantes masculinos expresaron su preocupación por la disminución de rendimientos y por los altos costos de producción.

El problema que tenemos es que ponemos líquido y fertilizante, y si no lo ponemos no da la milpa. Nuestra milpa ya no es tan grande y bonita. (Manuel Jiménez, 47 años, Elambo Bajo).

Pero aun con la fertilización química, los rendimientos son muy bajos, porque "la tierra ya no da" (Pedro X., 27 años, Elambo Bajo).

Para algunas mujeres entrevistadas, el problema con la fertilización química no solamente consiste en el precio inaccesible y el agotamiento de tierras, sino que también hay repercusiones para la salud humana:

La gente de ahora utiliza puro fertilizante en cualquier cosecha, por causa de todo esto existe tanta enfermedad, hay mucha enfermedad, decimos que estamos enfermos y nosotros mismos lo buscamos (Participante en un taller en Nachig).

Como problemas en cuanto a la milpa, tanto hombres como mujeres mencionaron cambios climáticos, especialmente lluvias más tardías y menos regulares:

¿Cómo vamos a sembrar maíz, cómo vamos a sembrar las cosas, si ya no hay lluvia? (...) Antes llovía más fuerte, y daba más seguido. Ahora en medios de mayo empieza a llover (María Hernández, 40 años, Zinacantán).

A partir de la década de 1980, ocurrió una nueva transformación al extenderse la floricultura en detrimento del cultivo de la milpa. En la micro región florícola, que abarca entre otros lugares Zinacantán y Nachig (Díaz-Coutiño, 1998), hubo una expansión fuerte de la floricultura bajo invernadero. Este sistema 
de producción es intensivo en el uso de agua y en el empleo de agroquímicos.

En el presente la floricultura provee de ingresos monetarios a muchas familias de Zinacantán. Existen perspectivas contrarias acerca de la floricultura. Por un lado están las personas cercanas a esta actividad, quienes tienen - como no es de sorprender- posturas más bien favorables:

La razón de los invernaderos es que aquí sembrando maíz no nos da para comprar grandes cosas, simplemente nos da para alimentarnos, para un sustento de la familia, pues. En cambio con estos invernaderos allí se producen cantidades, y a poco tiempo. El maíz, creo que es seis meses o más que necesita, en cambio en el invernadero pones y en tres meses ya estamos cosechando. Y hay más entrada de dinero. Por lo mismo estamos cambiando a veces el maíz por flores (Margarita, 26 años, hija de floricultor, Zinacantán).

Si bien no son ajenos, tampoco identifican como muy graves los problemas para la salud humana y para el ambiente causados por la floricultura: "No uso guantes ni cubrebocas. Yo nunca me he enfermado, pero sí he escuchado de personas que les salen ronchas en la piel" (Julio, 23 años, Zinacantán).

Por otro lado, las personas que no dependen de la floricultura subrayan más los efectos negativos que este cambio ha generado, sobre todo la contaminación por agroquímicos:

Tanto fumigar, tantas insecticidas, eso es lo que hace mal. A veces con el olor de insecticidas que están fumigando, hasta dolor de cabeza da. Hasta mareos da. Porque hay unos invernaderos que están muy cerca. A veces está uno comiendo algo, alguna fruta, y ya llega el olor, ya se siente bien feo. Allí a veces nos podemos enfermar, porque olemos el olor, estamos respirando ya olores, (María Hernández,
40 años, vive en la orilla de Zinacantán donde hay muchos invernaderos).

Hay tres consecuencias negativas que los colaboradores de Zinacantán, no floricultores, perciben. La primera es la contaminación por agroquímicos. Una segunda consecuencia es que "los invernaderos acaban con el bosque", porque por un lado se necesita madera para la construcción de invernaderos y por otro lado los invernaderos están empujando el límite del bosque más afuera de la comunidad y arriba hacia las montañas circundantes. Una tercera consecuencia es el uso de gran cantidad de agua, en claro contraste con la escasez del vital líquido para el consumo humano.

Loscambios en el cultivo de la milpay, especialmente, la pérdida de fertilidad de suelos y los altos costos de producción, fueron referidos por los hombres en Elambo, Nachig y Jech ch'entik, mientras que el cambio de milpa hacia la floricultura fue mencionado por ambos sexos en Nachig y Zinacantán cabecera. Fue en esta última localidad en donde hubo una crítica masiva de mujeres hacia el empleo de agroquímicos y el uso del agua por los floricultores.

\section{Cambios en torno al agua}

La situación en cuanto al acceso al agua que enfrentan los habitantes de los cuatro poblados es bastante diferente en términos de cantidad y calidad. De esta situación dependen las perspectivas de los lugareños en relación con el líquido vital. Clave en el tema del agua es el abasto y acceso, o sea la manera de cómo las personas se pueden proveer de este bien indispensable.

Mientras que Elambo, una comunidad alejada y marginada, tiene agua entubada desde la década de 1970 y lo celebra todavía como una gran ventaja - aunque ahora ya no es suficiente-, la gente de Jech ch'entik todavía no cuenta con agua entubada y pocos tienen la posibilidad financiera de comprar agua en pipa. Allí la problemática del agua se entrevera con los conflictos 
políticos que se viven dentro y fuera de la comunidad. En Nachig y en Zinacantán el abastecimiento de agua potable por tubería es relativamente garantizado aunque no libre de disputas-, y muchas personas complementan el agua entubada con agua de pozos o manantiales privados. Pero en estas dos comunidades preocupa que se estén secando las fuentes de agua que antes había dentro y alrededor de los pueblos, porque ya no llueve como antes.

En la cabecera los informantes no floricultores ponen énfasis en la floricultura como causante de escasez y posible contaminación del agua. Tanto los informantes como la literatura revisada reportan conflictos en torno a ciertos manantiales entre floricultores, horticultores y los pobladores (Burguete, 2000: 200), ya que los primeros se adueñaron de los manantiales y empezaron a captar el agua directamente desde los ojos de agua, con la consecuencia de que los arroyos quedaron secos o con muy poca corriente.

iAhora, Dios! Vas ahora al río para lavar, nada de agua. Porque ya son muchos los que siembran flor, puro invernadero. Por eso ya no hay agua. Antes el agua se veía bonita, azul; no se veía la basura, las bolsas de jabón se las llevó el río. Ahora, ay Dios, botellas de esos que usan los insecticidas y todos los que trabajan allí. Está estancado en los charquitos de agua, porque ya no hay mucha agua ya (María Hernández, 40 años, Zinacantán).

En todas las comunidades se mencionaba además el crecimiento de la población: antes sí alcanzaba el agua, pero ahora, con tanta gente, ya no es suficiente para todos. Con mucha frecuencia se menciona que ya no llueve como antaño a causa de la deforestación y que por eso ya no hay suficiente agua.

En donde hay conflictos religiosos, se atribuye la escasez de agua - por parte de los católicos tradicionales - al cambio en las costumbres. Mientras que los católicos tradicionales siguen celebrando sus fiestas alrededor del agua para agradecer al dueño de la tierra y para pedir que venga la lluvia, los evangélicos - y muchas personas jóvenes- ya no lo hacen.

Desde antes ha quedado que hay yacimientos de agua que los tenemos que cuidar y que tenemos que respetar. Pero ahora que ya no hay nada, los evangélicos ni rezan ni nada, pues se está acabando todo y la tierra ya está seca. Pero ahora ya he visto que ya hay pozos secos de los evangélicos, porque ellos ya no les ponen cruces ni velas, pues los pozos ya están secos y ya no hay nada (María Pérez, 55 años, Nachig).

A parte de la escasez, en todas las comunidades se mencionó la contaminación del agua como un problema grave ya que trae consigo enfermedades si es usada para el consumo humano, hay contaminación visual y la proliferación de mosquitos. Mientras que en la cabecera algunas mujeres atribuyeron esta contaminación sobre todo a la floricultura y al empleo de agroquímicos; en Nachig, Elambo y Jech ch'entik mencionaron la basura y las heces fecales como fuentes de contaminación -en estas últimas dos comunidades no hay recolección de basura, y en ninguna de las tres hay drenaje.

Lo que quiere es que cada una de nosotras tengamos consciencia de tener limpio nuestro planeta, agua limpia, en cambio ahora aunque tengamos pozo nosotras mismas lo contaminamos por las letrinas que tenemos cerca. Lo que yo sugiero es que hubiera drenaje para que la suciedad vaya lejos, en mi observación veo antihigiénico como estamos viviendo (Juana López, 35 años, Nachig).

Las dificultades en cuanto al acceso y la calidad del agua fueron relatadas en todas las localidades y por todos los participantes. Hay una cierta diferencia entre hombres y mujeres: si bien ambos ven con preocupación los problemas que tienen con el agua, las mujeres hacen más 
énfasis en cómo conseguir el líquido elemento — “antes cargábamos, ahora viene del tubo y de la pipa"-, y en su uso doméstico.

Pues yo veo que está acabándose el agua, como nosotros ya no tenemos agua en la comunidad. Pero me imagino que los hombres no sufren porque ellos solo se cambian y dejan sus ropas sucias, pero las que más sufrimos somos las mujeres porque vamos en busca de agua, aunque ahora hay personas que compran agua en pipa porque todavía hay. Pero si se acaba y sigue acabándose el agua iqué vamos a hacer? Aunque tengamos mucho dinero, pues, iqué podemos hacer? (María Pérez, 55 años, Nachig).

No encontramos diferentes perspectivas en cuanto al agua entre generaciones. La diferencia más notable se da entre religiones, ya que algunos católicos culpan a los evangélicos por la falta de agua, y los evangélicos a los católicos porque les limitan y hasta anulan el acceso al agua por discriminación religiosa.

Se nota generalmente un patrón de divisiones comunitarias o conflictividad en las cuatro comunidades: floricultores versus no floricultores, evangélicos versus católicos tradicionales.

\section{Cambios en los bosques}

En el municipio de Zinacantán, como en Los Altos de Chiapas en general, dominaron originalmente bosques deciduos de pino encino. Las personas entrevistadas mencionaron sobre todo diferentes especies de encino que hubo antes: tulan (Quercusrugosa), chikinib (Q. castanea, 2. crispipilus), batsite (२.crassifolia)', diferentes especies de pinos (Pinus spp.), así como madroño (Arbutusxalapensis), y algunas especies de menos importancia.

Aparte de los árboles para leña — sobre todo las diferentes especies de Quercus - y para madera (Pinus spp.), se utilizaba una gran variedad de recursos forestales como hongos y plantas comestibles, plantas de ornato y medicinales, y animales silvestres comestibles. El uso y el conocimiento de estos recursos variaron según edad, sexo y localidad, siendo las mujeres ancianas las que más recursos del bosque en general mencionaron y los hombres jóvenes los que menos los conocen.

En todas las localidades, tanto hombres como mujeres de diferentes generaciones, describieron un cambio muy notorio en los bosques, tanto en la abundancia de árboles como en la diversidad. Los cambios en los bosques fueron mencionados por la gente como "ya no hay árboles", "se están acabando los bosques", "están talando mucho", "ya no crecen grandes", o por los entrevistados jóvenes como "deforestación".

Existe una percepción diferenciada acerca de la fecha en que inició la deforestación. En Elambo las personas jóvenes todavía recordaban el lugar con mucho bosque y con árboles grandes. Según los informantes, aproximadamente en los últimos quince años se empezó con la tala masiva de árboles grandes, debido en parte a la introducción de la motosierra.

En Jech ch'entik y en Nachig las personas mencionaron una deforestación desde hace aproximadamente veinticinco años, más o menos coincide con el principio de la floricultura. Se tumban árboles para edificar los invernaderos en lugares boscosos y se utiliza la madera para la construcción de los mismos. Como se tienen que renovar los invernaderos de madera por lo menos cada diez años se vuelven a cortar árboles para el invernadero, aun cuando ya esté establecido.

En Zinacantán cabecera el cambio de los bosques inició hace mucho tiempo, aproximadamente cuarenta años o más, mucho antes de la introducción de la floricultura. Solo las personas muy mayores recuerdan todavía cómo era el bosque antes en ese lugar y adjudicaron la pérdida del mismo al crecimiento demográfico y el avance de la mancha "urbana". Doña Magdalena, de 74 años, comenta cómo 
en su niñez los árboles eran grandes y fuertes, pero ya hace aproximadamente 40-50 años había cerros casi deforestados, porque de allí la gente local y las personas que venían para cumplir sus cargos religiosos habían agarrado leña. Doña María, su hija, recuerda que ya había muy pocos árboles cuando ella creció.

Cuando yo era chiquita, no había, ahora hay más, porque ya volvieron a sembrar los cipreses, pero antes creo que crecieron mucho el roble, en onte', el chiquinib, como me decían. Yo vi esos árboles chiquitos, creo que tumbaron todo para la leña (María de la Cruz, 47 años, Zinacantán).

Con la aparición de los invernaderos y la expansión de las áreas de cultivo de flores, así como con el crecimiento urbano, la deforestación empezó a acelerarse y los bosques fueron reducidos notoriamente en las montañas circundantes.

En los cerros muy afectados por la deforestación alrededor de Zinacantán, se reforestó hace aproximadamente veinte años, pero en vez de resembrar con encinos, especie nativa, se reforestó con ciprés y pinos, árboles muy poco apreciados y de poca utilidad para los habitantes. González et al. (2007: 13) describen como el "incremento de Pinus spp. está asociado con una reducción en la riqueza de bejucos, lianas, arbustos y árboles del interior" y un empobrecimiento florístico de los bosques. En los bosques restantes alrededor de Zinacantán es raro encontrar encinos y otros árboles caducifolios, así que los bosques allí se convirtieron casi por completo en bosques de coníferas. Por lo tanto, no ha de sorprender que el joven Juan Alex recuerde únicamente el bosque de coníferas.

\section{- ¿Cómo ha cambiado el bosque desde que tú naciste?}

- Como te digo, no ha cambiado tanto. Tal vez mi mamá es la que vivió más el cambio de muy abundante a muy despoblado. Yo veo lo más común, el ciprés, el pino (Juan Alex González, 23 años, Zinacantán).
En otras comunidades hubo acciones de reforestación muy puntuales hace años, también sobre todo con cipreses y pinos, pero la gente menciona que ya hace mucho que no se siembran árboles en cantidades notables.

En todas las localidades, las personas señalan la presión demográfica como una causa importante para la deforestación. Con la llegada de nueva gente y la necesidad de los jóvenes de fincar en algún lugar, se cortan árboles para hacer espacio y construir nuevas casas y calles. En Elambo, la comunidad con más área de bosque dentro de las cuatro estudiadas, la presión demográfica y el cambio en el cultivo de la milpa están siendo percibidos como causas de la deforestación. Jacorzynski describe un diálogo que tuvo en Xulvo', comunidad de Zinacantán, en el cual se adjudica la pérdida de bosque al cambio en el cultivo de milpa, lo que nos muestra que el discurso de la deforestación en el municipio de Zinacantán no varía mucho y que hay interpretaciones parecidas en diferentes parajes (Jacorzynski, 2004: 244).

El cambio en los bosques era percibido claramente como un problema. Hombres y mujeres añoraban que antes, cuando hubo más árboles, era más bonito, el clima era más fresco, había más animales y plantas silvestres del bosque, y, sobre todo, era más fácil acceder a la leña, recurso imprescindible para la vida cotidiana en Zinacantán.

Mientras que antes todas las mujeres de las comunidades de Zinacantán iban a buscar leña a donde quisieran — con la regla de solamente levantar ramas caídas o eventualmente cortar ramitas de árboles-, conforme creció la presión sobre los bosques se hizo más escasa la leña y la gente empezó a cercar sus terrenos, o por lo menos a delimitarlos claramente.
Ahora ya es más difícil que antes agarrar leña, antes en donde quiera caían los bracitos de los árboles grandes, cuando había viento caía mucho, sin necesidad de cortar, solo lo levantaban nada más las mujeres. Y 
ahora ya no hay de esas (Magdalena Montejo, 45 años, Elambo).

Ahora la norma es que cada familia busque leña en su propio terreno, pero como los terrenos se están fragmentando más y más en general hay menos áreas boscosas, esto se vuelve aún más difícil. La consecuencia es que en las comunidades semiurbanas las familias más ricas utilizan estufas de gas y las que no pueden costear la estufa compran leña que viene de otros lugares. En las comunidades rurales, las mujeres siguen yendo por leña, pero con grandes dificultades para conseguirla. "Para agarrar leña, cada quien va en su parcela, es más seguro. Nos agarran los dueños y nos llevan al bote si nos vamos en otro terreno", dice Pedro (27 años) de Elambo.

Pero no sólo la presión demográfica en Zinacantán es la causa de la escasez de leña, también el hecho de que el bosque se convirtió en una mercancía, ya que se pueden vender parcelas enteras para leña o carbón, como ha ocurrido en Jech ch'entik y en Elambo.

Pues la verdad es muy difícil, porque solo algunos se vuelven ricos por vender los árboles, pero los más pobres somos los que sufrimos, porque ya no tenemos dónde conseguir la leña, (Señoras en un taller en Jech ch'entik).

\section{¿Cómo varían las perspectivas relativas a los cambios en los bosques?}

Comolas mujeres en Zinacantán tienen tradicionalmente la responsabilidad de ir al bosque a conseguir leña, no es de sorprender que sean ellas las que más conocimiento tienen sobre las diferentes plantas que hay. Las mujeres, y en especial las señoras adultas y ancianas, describieron una gran variedad de árboles en el bosque, los que hubo antes y los que todavía existen. Los señores adultos y ancianos también conocían los árboles, pero mencionaron menos especies diferentes y los hombres jóvenes casi no conocían los árboles del bosque. Se confirma el supuesto de la psicología ecológica que acción y percepción son inseparables y que percibimos diferentes oportunidades ambientales en función de las actividades en las que estamos involucrados. Las mujeres mayores fueron las que hicieron más énfasis en el problema de la leña, mientras que las jóvenes - a excepción de las de Elambo- parecen haberse acostumbrado a la idea de comprar leña o cocinar con gas. Tanto jóvenes como adultos y ancianos remarcan la deforestación como una pérdida, sin embargo el horizonte de tiempo con que lo hacen difiere de comunidad a comunidad.

Aparte de la leña, las personas entrevistadas mencionaron varias plantas comestibles, tanto del bosque como del río, que antes formaban parte de la dieta, pero que muchos y muchas jóvenes ya no las conocen o "no saben comer". Mientras que los hombres mantuvieron que las verduras siempre estaban cultivadas — "no crecen así nada más"-, las mujeres mencionaron varias especies de plantas silvestres: hierbamora (Solanum americanum), maruch itaj (Amaranthus hybridus) y napux (Brassica campestres), aparte de los hongos que antes, en época de lluvia, formaban parte importante de la dieta.

Pero, por diferentes razones, entre otros la deforestación y los cambios climáticos, la práctica de recolección de plantas silvestres casi se acabó. Una informante de Zinacantán comenta:

Se siente uno mal, porque estaba uno acostumbrado solo a cortarlo, ahora ya todo es comprado. Antes aquí cerquita había nabitos, en estos tiempos ya había muchas verduras, pero ahora ya nada (...) En el lugar de hongos, antes se conseguía todo una cubetita, bien sabroso. Pero ahora en el lugar de hongos es puro invernadero. Por eso ya no se consigue (María Hernández, 40 años, Zinacantán).

Comentarios parecidos hicieron las participantes en Nachig y en Jech ch'entik, en donde por la deforestación 
ya no encuentran flora comestible. En Elambo sigue vigente la práctica de recolección de hongos y plantas comestibles por parte de algunas mujeres, sin embargo, ésta se admite con cierta pena. Los y las jóvenes no practican la recolección de plantas, tanto las mujeres como los hombres jóvenes lo conocieron solo por lo que sus madres o abuelas les habían platicado.

Mientras que las mujeres tenían grandes conocimientos sobre las plantas silvestres y deploraron más su pérdida que los hombres, los animales silvestres fueron referidos por los dos sexos por igual. Aquí la diferencia era notoria entre las generaciones. Las y los jóvenes mencionaron como animales del bosque solamente a las ardillas, pájaros y conejos —y habían escuchado que antes había venados y otros animales-, no obstante las personas adultas y mayores recuerdan varios animales del bosque que antes había, pero ahora ya no o casi ya no, por ejemplo venado (Odocoileus virginianus), tlacuache (Didelphis virginiana), coyote (Canis latrans), tepezcuintle (Cuniculuspaca), zorro gris (Urocyon cinereoargenteus), comadreja (Mustela frenata), entre otros.

\section{Contaminación por desechos}

Los desechos sólidos inorgánicos fueron generalmente referidos como basura. En lo que se mencionó como tal destacaron las botellas de refresco, bolsas de nailon, pilas, y las llamadas "bolsas de Sabritas". Según algunos participantes, empezó a haber basura aproximadamente hace 20-30 años. Esta visión se debe a que los desechos orgánicos que siempre había, no están percibidos como basura o no como un problema digno de mencionarse, ya que se desintegraban. A muchas personas se les hizo difícil estimar el tiempo desde cuándo había presencia de basura en el paisaje ya que los cambios se fueron dando de manera paulatina. Los y las jóvenes ya no recuerdan un tiempo sin basura inorgánica. Para ellos siempre hubo, pero saben que antes la gente tenía otros hábitos de consumo y suponen que por ende no hubo tanto desecho inorgánico.
Los desechos mencionados arriba son muy visibles en calles, patios, terrenos, cuerpos de agua y bosques de las comunidades de Zinacantán. Que la basura esté regada en todos estos lugares está percibido como un problema - denominado contaminación, por algunosya que según los colaboradores huele mal, se ve feo, provoca moscas y trae enfermedades. Como causas de la contaminación por basura, los colaboradores hombres y mujeres de todas las edades- mencionaron que el consumo ha cambiado, y que ahora todo viene envuelto o en bolsas de plástico. Además se mencionó que el crecimiento poblacional agudizó el problema de la contaminación.

El problema se percibió en todas las comunidades, aunque se hizo más énfasis en donde no hay recolección de basura: en Jech ch'entik y Elambo, y se mencionó el tema de la basura asociado con el tema del agua.
Ahora en todas partes hay basura, sea calle o agua, está llena de basura. Pero lo malo es que no tenemos agua, y cuando ya no conseguimos agua que tomar, pues la tomas, aunque tenga basura (...) Es que no entendemos que debemos cuidar el agua y no ponerle basura (Señora en un taller en Jech ch'entik).

En las comunidades donde no hay servicio de recolección de basura, las familias - y allí generalmente las mujeres - tienen que deshacerse de los desechos de alguna manera. Lo más común es quemar todo lo que se puede quemar: plásticos, papel, desechos orgánicos secos, etcétera. Los desechos que no se pueden quemar - latas de aluminio y fierro, vidrio, pilas, etcétera - se entierran o se echan en algún barranco.

En Nachig y la cabecera de Zinacantán hay servicio de recolección de basura, y eso ciertamente está experimentado como un gran alivio:

Antes había mucha contaminación, pero como ahora ya tenemos camión de volteo para recolectar la basura, quiere que juntemos la basura cada uno de nosotros 
en nuestro patio de la casa, no es igual como antes que nunca terminábamos de quemar basura (Señoras en un taller en Nachig).

Aun así el problema de la contaminación por basura persiste, ya que muchas personas continúan tirando sus desechos en un barranco, o en la calle.

Fue interesante observar cómo las personas percibían la contaminación en espacios públicos a diferencia de los espacios privados. Los espacios públicos eran percibidos como muy contaminados, mientras que casas y patios se encontraban más limpios. "Antes había mucha basura alrededor de nuestra casa porque no teníamos higiene", comenta una señora mayor de la cabecera de Zinacantán. En las pláticas del programa Oportunidades y de las Clínicas de Salud, adonde asisten la mayoría de las señoras entrevistadas, se pone mucho énfasis en no acumular basura ni otros focos de contaminación en las casas y patios. "Los de Oportunidades ya no nos permiten basuras ni animales" (María Hernández, 40 años, Zinacantán).

Mientras que los llamados a mantener limpias las casas y patios — siendo esto responsabilidad única de las mujeres - parecían tener frutos, los llamados generales a no tirar basura o "poner la basura en su lugar" no eran muy efectivos, como se constata en esta declaración:

Es que la gente, pues, ordenamos que no tiren basura, que no tiren pañales, que no tiren plástico, que no tiren todo, pero la gente aquí no quiere. Nada. Entonces, un rato lo hablamos en público, y la otra semana ya lo tiran otra vez. A veces tiran animales, de todo... (Andrés Pérez, 48 años, Jech ch’entik).

Sobre las soluciones al problema de la basura, hay posturas diferentes. En Elambo y Jech ch'entik decían que se necesitaban limpiar las áreas contaminadas y el ojo del agua. De esta forma sí había consenso sobre qué hacer después con la basura. En Nachig y Zinacantán proponían poner botes en el centro del poblado para que la gente echara allí sus desperdicios, juntar la basura y entregarla al carro recolector para que fuera trasladada al tiradero comunal. Parecido al problema de la contaminación del agua por heces fecales - "que la suciedad vaya lejos"-, la solución que algunos proponían era "juntar y tirar lejos" o "juntar y quemar", como si la basura dejara de contaminar por el simple hecho de no ser visible en nuestro ambiente inmediato. Los jóvenes del telebachillerato mencionaron la idea de reciclar y concientizar sobre el problema de la basura:

Para enfrentar el problema hay que impartir pláticas en la cabecera municipal de Zinacantán, en las escuelas, con los padres de familia, hay que poner carteles en lugares descuidados, tener más cuidado de no tirar. Y otra cosa es reciclar, hay que reciclar el plástico y todo (Jóvenes en un taller en la escuela telebachillerato de Zinacantán).

Algunas señoras en las comunidades mencionaron la posibilidad de reducir la basura con acciones tan sencillas y concretas como por ejemplo traer el chal para comprar huevos y llevar una servilleta cuando van a la tortillería.

Ambos sexos percibían la existencia de la basura como un gran problema y nadie tiene una solución patente. Las mujeres hicieron más énfasis en las maneras de cómo deshacerse de los desechos - por ejemplo quemar, enterrar, tirar lejos, etcétera- que los hombres, ya que por la división del trabajo existente, a ellas les toca diario realizar esta tare $\mathrm{a}$.

En el tema de los desechos destacó que hay percepciones similares acerca de la gravedad y el origen del problema entre hombres y mujeres, entre las generaciones y entre las localidades. La única diferencia encontrada fue que las personas jóvenes manifestaron explícitamente que ellos y ellas mismas - o sea, nosotros mismos - generamos la basura y somos los responsables de la contaminación. Desde 
esta postura se están empezando a buscar alternativas concretas, aunque sean iniciales, como reciclar o reducir. Sin embargo, otro tipo de enunciados comunes fueron: "la gente no entiende", "la gente de afuera tira mucha basura", etcétera.

\section{Diferencias entre las perspectivas de los cambios ambientales}

Los cambios no son percibidos y expresados de la misma manera por los sujetos; es decir, las perspectivas que los lugareños tienen dependen de muchos factores, entre ellos la edad, el género, el estatus socioeconómico, la fuente de ingresos, la religión y las condiciones materiales de la comunidad en donde viven. A continuación sintetizamos las diferencias de las perspectivas ambientales entre las localidades, entre los géneros y entre los diferentes grupos de edad.

\section{Diferencias entre parajes}

Encontramos diferencias en las perspectivas ambientales entre los pobladores de los parajes estudiados, en ciertos temas eran más notorios que las diferencias entre géneros y generaciones. Los y las participantes hicieron un énfasis diferente en los problemas ambientales percibidos y vividos por ellos en las diferentes localidades (ver Cuadro 5). En Zinacantán cabecera los temas principales eran la escasez de agua, la contaminación por plaguicidas, y el deterioro de los bosques; en Nachig, el tema prominente era la alimentación, enfermedades resultantes de ella, y conflictos por agua; mientras que en Jech ch'entik los participantes hicieron énfasis en los problemas que tienen con la escasez de agua y leña y con la contaminación por basura. En Elambo enfatizaron mucho la contaminación por basura y los problemas con la milpa, especialmente la pérdida de fertilidad de la misma.

Las diferencias entre parajes se explican en el marco de las diferencias productivas y ambientales, ya que la fuente principal del ingreso, los patrones de consumo, los servicios y la disponibilidad y calidad de recursos naturales, no son los mismos en todos los parajes. Esto sugiere que más allá de las experiencias individuales, los factores determinantes en la conformación de sus perspectivas ambientales son las vivencias que tienen las personas con su entorno.

\section{Diferencias entre hombres y mujeres — condición de género}

Dentro de los parajes se notaron diferencias entre hombres y mujeres en cuanto a la importancia que atribuyen a ciertos problemas ambientales: dentro de cada comunidad, las mujeres enfatizaron más los temas de la leña, el agua y la alimentación. En cambio, los hombres generalmente se preocuparon por los cambios productivos y la pérdida de fertilidad de la milpa.

Desde la perspectiva Ecofeminista, gran parte de las actividades de subsistencia están íntimamente relacionadas con los recursos naturales (Mies, 2004). Un deterioro de los recursos naturales implica cada vez más dificultades para llevar a cabo actividades de subsistencia. Muchas de estas actividades, como conseguir leña para cocinar, acarrear agua, recolectar plantas comestibles y criar animales de traspatio, las llevan a cabo las mujeres en Zinacantán y, por ende, ellas estarían siendo más afectadas por el deterioro de ciertos recursos naturales —o estaban afectadas, dependiendo de la comunidad y la edad, ya que las mujeres jóvenes en las comunidades semiurbanas casi ya no acarrean leña ni agua, ni recolectan plantas comestibles y muy pocas crían animales de traspatio.

Desde la línea Mujeres y Medio Ambiente se enfatiza que, debido a sus actividades en la esfera reproductiva en el ámbito doméstico, las mujeres tienen un conocimiento especial acerca de una parte de los recursos naturales, por ejemplo el bosque y las plantas alimenticias, así como un interés especial en su manejo sustentable (Joekes, 2004:500). Por su parte, los 
hombres centran su interés en otros recursos naturales, por ejemplo los terrenos para el cultivo y la milpa. Esta línea mantiene que la división sexual del trabajo es central para el manejo, los conocimientos y el interés en los recursos naturales, hecho que se vio confirmado en varias ocasiones en este estudio.

La línea Género y Medio Ambiente resalta que por las relaciones desiguales de género existen papeles distintos frente al ambiente entre hombres y mujeres. Éstas pueden implicar intereses diferentes y conflictos por el control y manejo local de los recursos (Joekes, 2004: 498; Paolisso, 1998: 200).

Lo que se ejemplifica en este estudio, cuando en Jech ch'entik las mujeres condenaron la venta de terrenos para deforestación, mientras que los hombres justificaron ese mismo hecho por la necesidad de ganar dinero. Otro ejemplo son los conflictos por el acceso a aguas superficiales entre algunas mujeres que necesitan los ríos para lavar ropa, por un lado; y los floricultores, en su mayoría hombres, por el otro. Un aspecto importante destacado por esta teoría es que las prioridades no solamente pueden diferir entre hombres y mujeres, sino también al interior de los hogares y al interior de las comunidades, o sea que el género se articula con la edad, la posición socioeconómica, la identidad religiosa, étnica, etcétera. Eso quedó claramente comprobado en este estudio, ya que en algunos temas la edad, la ocupación, la religión y la comunidad de proveniencia, parecen más importantes que la pertenencia a un género u otro, aunque éste siempre se encuentra presente y transversal en las demás diferencias.

\section{Diferencias entre jóvenes, adultos y ancianos}

Las perspectivas de jóvenes, adultos y ancianos acerca del deterioro de los recursos naturales son diferentes, ya que los y las jóvenes simplemente no identifican ciertos cambios que los adultos o los ancianos reconocen, por ejemplo en cuanto a alimentación, bosques, agua, o en cuanto al ámbito productivo. Eso es entendible si consideramos que una gran parte de cambios ambientales - y socioeconómicos - iniciaron en Zinacantán hace aproximadamente 25 a 30 años, tiempo en el cual los jóvenes eran todavía bebés o infantes, por lo que ellos no tienen una referencia real y vivida de como era antes.

También fue notorio cómo las personas jóvenes ya se habían "acostumbrado" al nuevo estilo de vida, por ejemplo, para las mujeres jóvenes es normal cocinar con gas o comprar leña, mientras que las adultas y ancianas deploran la escasez de leña. Esto parece estar ligado a nuevas formas de producción y consumo: se produce para poder comprar los bienes necesarios, ya no para el autoconsumo.

Los jóvenes participantes identificaron algunos problemas ambientales que para los ancianos no eran tan urgentes, como el problema de la basura, en donde los primeros hicieron énfasis en soluciones técnicas, organizativas y de educación: reciclar, organizar al pueblo, concientizar.

Las diferencias entre las generaciones se deben por un lado al tiempo vivido de referencia, y por otro lado a valores y estilos de vida divergentes, seguramente en parte influenciadas por la modernidad, misma que llega a través de la educación escolar, los medios de comunicación, y por el contacto con personas de afuera. Son los jóvenes los que asumen esta modernidad más claramente en su forma de secularización, descubrimientos científicos, avances técnicos y educación, e interpretan los cambios ambientales bajo esta lógica.

\section{Conclusión}

El concepto de perspectivas ambientales puede ser útil para dar cuenta de los intereses diferenciados que tienen hombres y mujeres, jóvenes, adultos y ancianos, y sus explicaciones y valoraciones de los cambios, ya que las perspectivas ambientales recogen la acción/ percepción de las personas y su interpretación. Como 
mujeres y hombres tienen papeles bastante distintos por su condición de género, sus actividades son diferentes y las interpretaciones resultantes de esas también. Pero más allá de la diferencia de género que es transversal a todas las demás diferencias- el concepto permite ver otras variables importantes. Las experiencias directas de personas provenientes de diferentes lugares con condiciones ambientales disímiles son igualmente desiguales, por ejemplo si provienen de lugares urbanos versus rurales, de comunidades con servicios e infraestructura versus de poblados rodeados de bosques: las personas de cada esfera se mueven dentro de ambientes que brindan otras cosas. También el cambio vivido e interpretado por ancianos, adultos y jóvenes, difiere según sus conocimientos prácticos, sus habilidades y la cultura dentro de la cual están inmersos.

Concluimos que el concepto es útil para analizar cómo personas de un mismo grupo cultural perciben e interpretan su ambiente y los cambios que están ocurriendo en él, y entender por qué hay diferentes visiones en un grupo supuestamente homogéneo. Sin embargo, creemos poder concluir a partir del trabajo empírico que no solamente las "vivencias individuales" resultan de suma importancia en la conformación de las perspectivas ambientales, sino también hay que tomar en cuenta las vivencias colectivas de personas con características parecidas, por ejemplo hombres, mujeres, ancianos, adultos, jóvenes o personas de una determinada comunidad, ya que cada grupo de estos comparte actividades, roles o condiciones específicas.

A pesar de las diferencias encontradas en los participantes, hay problemas comunes que todos y todas identificaron y sobre los cuales hay que trabajar conjuntamente. Estamos convencidos de que en el caso de Zinacantán los obstáculos para enfrentar los cambios ambientales no están en las posiciones diferentes de las personas, sino en las divisiones políticas, así como en la falta de iniciativas locales.
Creemos que es necesario investigar más acerca de las divisiones comunitarias y cómo éstas pueden afectar el ambiente biofísico natural, tema que se toca aquí solamente de manera parcial. Las reflexiones sobre divisiones comunitarias se reducen muchas veces a cuestiones de partidos políticos y de religiones, y se enfatiza la violencia que han desatado, tema que ciertamente es muy impactante y es importante denunciar. Es tiempo de enfocar el efecto que tienen estas divisiones en el manejo y la protección de recursos naturales.

Para futuras investigaciones vemos también la necesidad de indagar más sobre las diferencias entre generaciones. Teniendo los y las jóvenes visiones a menudo diferentes que los adultos y ancianos, sería sumamente importante entender a qué se deben estas diferencias, cómo es que los jóvenes se imaginan un manejo sustentable de los recursos naturales y cuál podría ser el puente con las experiencias y conocimientos que sus padres/madres y abuelas/abuelos tienen.

Los cambios ambientales detectados en este estudio, como la deforestación, escasez y contaminación del agua, los problemas con los agroquímicos y problemas relacionados con el cambio en el consumo y los desechos, son comunes en muchas partes del mundo, en lugares muy lejanos entre sí. Sin embargo, hay acciones concretas que se pueden llevar a cabo en cada lugar para contrarrestar estos cambios, algunos de los que ya emprenden las señoras pertenecientes a Mujeres Sembrando la Vida, como reforestación, uso de estufas ahorradoras de leña, construcción de letrinas secas, y muchos otros que hay que iniciar no solamente en este grupo.

Esperamos que este trabajo sea útil para la reflexión y toma de acción de los propios sujetos, para la intervención de organizaciones sociales y civiles, y tal vez hasta para el diseño de políticas públicas en cuanto a equidad, desarrollo y conservación de los recursos naturales. Las propios habitantes de Zinacantán son los 
agentes de cambio indicados, son ellos y ellas quienes pueden y deben hacer las reivindicaciones necesarias, y son quienes pueden cambiar las acciones, tanto propias como las de sus gobiernos.

\section{Agradecimientos}

A Xunka Hernández por su valioso apoyo, tanto en la cofacilitación de los talleres, como en las entrevistas y en la traducción.

A las señoras de Mujeres Sembrando la Vida por sugerencias importantes al inicio de esta investigación y por estar dispuestas a participar.

A todas las mujeres y hombres de Zinacantán, quienes dieron su palabra en entrevistas y talleres, por su apertura, su confianza, por las discusiones "calientes", y por compartir sus preocupaciones y esperanzas.

A todas las personas quienes participaron en los talleres, por su emoción, a través de la reflexión y las discusiones grupales.

A Marcelina Rodríguez, por su cuidadosa traducción y transcripción de las entrevistas y de las discusiones en talleres del tsotsil al español.

\section{Notas}

"Aquí distinguimos entre "semiurbano" y "rural" según la infraestructura disponible en la localidad: pavimentación de calles, oficinas de gobierno, servicio de salud, comunicación a través de carreteras, comercios, escuelas medias superiores.

\section{Bibliografía}

Arizpe, L. et al. (1993), Cultura y Cambio Global: Percepciones Sociales sobre la Deforestación en la Selva Lacandona, México: CRIM-UNAM/Miguel Ángel Porrúa.

Benez, Mara Cristina (2008), "Percepciones de la Calidad y de la gestión de las aguas superficiales de la cuenca de San Cristóbal de Las Casas, Chiapas", Tesis doctoral, México: Ecosur.

Burguete Cal y Mayor, Araceli (2000), Agua que nace y muere. Sistemas normativos indígenas y disputas por el agua en Chamula y Zinacantán, Científica 2, San Cristóbal de Las Casas: Proimmse-IIA-UNAM.

Chávez Ballado, Gloria (2007), "Percepción del ecosistema por la comunidad de San Crisanto en Yucatán de acuerdo con su actividad", en Cuicuilco, México: Escuela Nacional de Antropología e Historia, vol. 14, núm. 29, enero-abril 2007, pp. 99-114.

Collier, George A. (1992), "Búsqueda de alimentos y búsqueda de dinero: cambios en las relaciones de producción en Zinacantán, Chiapas", en C. Hewitt de Alcántara (comp.), Reestructuración económica y subsistencia rural, México: El Colegio de México/ UNRISD, pp. 183-221.

Collier, George A., David C. Mountjoy y Ronald B. Nigh (1994), "Peasant Agriculture and Global Change. A Maya response to energy development in southeastern Mexico", en BioScience, vol. 44, núm. 6, pp. 398-407.

Durand, Leticia (2008), "De las percepciones a las perspectivas ambientales. Una reflexión teórica sobre la antropología y la temática ambiental", en Nueva Antropología, Revista de Ciencias Sociales. Ambiente y Cultura, núm. 68, Enero-Junio 2008, pp. 75-87.

González-Espinosa, M. et al. (2009), "Tendencias y proyecciones del uso del suelo y la diversidad florística en los Altos de Chiapas, México", en Investigación ambiental. Ciencia y política pública, vol. 1, núm. 1.

Hartigan, Pamela (1998), Género, Ambiente y Salud. Incorporación de un enfoque de género en el trabajo de salud ambiental, División de Salud y Desarrollo Humano. Organización Panamericana de la Salud.

Hope, Anne y Sally Timmel (1995), Training for Transformation. A Handbook for Community Workers. Book I., Kleinmond, South Africa: Training for Transformation Institute. 
Ingold, Tim (1997), "Culture and the perception of the environment", en E. Croll y D. Parkin, Bush Base: Forest Farm. Culture, environment and development, Londres y Nueva York: Routledge, pp. 39-56.

Instituto Nacional de Estadística y Geografía, 2002. Anuario Estadístico de Chiapas.

Instituto Nacional de Estadística y Geografía, 2010, Censo poblacional. http://www.censo2010.org.mx/ [junio de $2011]$.

Jacorzynski, Witold (2004), Entre los sueños de la razón. Filosofía y antropología de las relaciones entre hombre y ambiente, México: CIESAS/Miguel Ángel Porrúa.

Joekes, Susan et al. (2004), "La integración del género en la investigación y las políticas ambientales", en Vázquez V. y M. Velásquez (comps.), Miradas al Futuro. Hacia la construcción de sociedades sustentables con equidad de género, México: PUEG/CRIM-UNAM/ COLPOS/IDRC, pp. 487-564.

Lazos, Elena y Luisa Paré (2005), Miradas indígenas sobreuna naturaleza entristecida. Percepciones del deterioro ambiental entre nahuas del sur de Veracruz, México: UNAM/Plaza y Valdés.

Martínez Corona, Beatriz (2000), Género, Empoderamiento y Sustentabilidad. Una experiencia de microempresa artesanal de mujeres indígenas, México: Grupo Interdisciplinario sobre Mujer, Trabajo y Empresa, A. C.

Mies, María (2004), "La necesidad de una nueva visión: la perspectiva de la susistencia”, en Vázquez V. y M. Velásquez (comps.), Miradas al Futuro. Hacia la construcción de sociedades sustentables con equidad de género, México: PUEG/CRIM-UNAM/COLPOS/IDRC, pp. 95-125.

Paolisso, Miguel (1998), "Avances de la investigación sobre género y medio ambiente", en Rivera Izábal, L. (coord.), Género y medio Ambiente, Cuernavaca, México: CIDHAL, Centro para mujeres. Programa Documentación, Educación y Cultura. Centro de Documentación "Betsie Hollants", pp. 199-208.

Rivera Izábal, L. (coord.) (1998), Género y medio Ambiente. Cuernavaca: CIDHAL, Centro para mujeres, Programa Documentación, Educación y Cultura.

Tuñón Pablos, Esperanza (2000), "La categoría género: discusión, preguntas y nuevas propuestas", en Léxico de Política, México: UNAM/FLACSO.

Vázquez García, Verónica (2003), “La gestión ambiental con perspectiva de género. El manejo integrado de ecosistemas y la participación comunitaria”, en Gestión y Política Pública, II semestre, vol. 12, Centro de Investigación y Ciencias Económicas, CIDE, pp. 291322.

Vázquez García, Verónica y Margarita Velázquez Gutiérrez (comp.) (2004), Miradas al Futuro: Hacia la construcción de sociedades sustentables con equidad de género, México: UEG/CRIM-UNAM/COLPOS/IDRC. 
Anexo: Cuadros y figuras

Figura 1: Ubicación de los lugares de estudio sobre perspectivas ambientales en Zinacantán, Chiapas

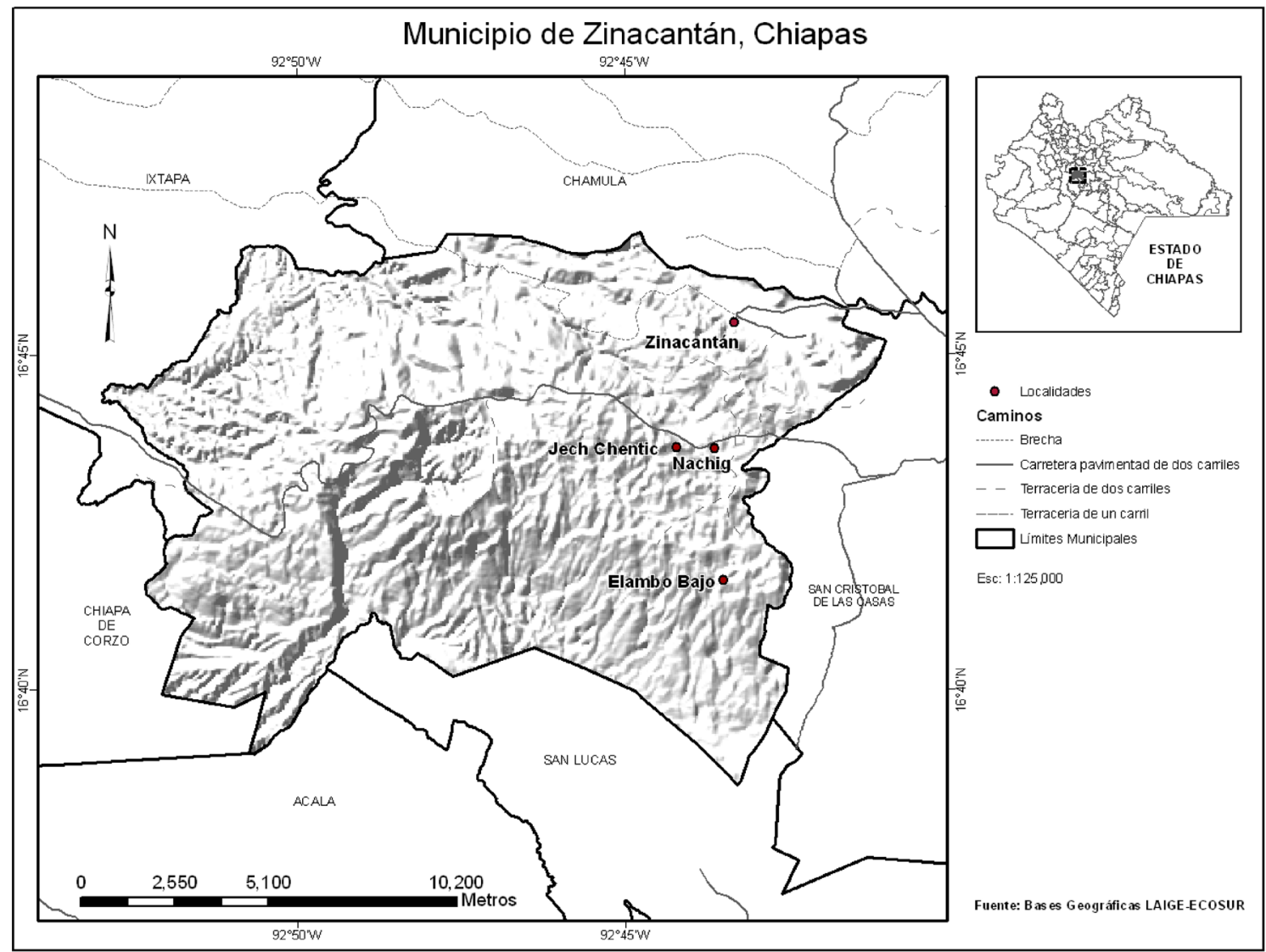

Fuente: LAIGE -ECOSUR. 
Cuadro 1: Diferencias y similitudes entre los lugares de estudio: número de habitantes y viviendas, infraestructura y condiciones ambientales

\begin{tabular}{|c|c|c|c|c|}
\hline & $\begin{array}{l}\text { ZINACANTÁN, } \\
\text { CABECERA } \\
\text { MUNICIPAL }\end{array}$ & NACHIG & JECH CH'ENTIK & ELAMBO BAJO \\
\hline Urbano/rural & Semiurbano & Semiurbano & Rural & Rural \\
\hline No. de habitantes & 3,876 & 3,260 & 892 & 629 \\
\hline No. de viviendas habitadas & 817 & 607 & 155 & 140 \\
\hline Conexión carretera/terracería & Carretera & $\begin{array}{l}\text { Carretera } \\
\text { panamericana }\end{array}$ & $\begin{array}{l}\text { Carretera } \\
\text { panamericana }\end{array}$ & $\begin{array}{l}\text { Terracería, } \\
\text { recientemente } \\
\text { parcialmente pa- } \\
\text { vimentada }\end{array}$ \\
\hline Actividad económica principal & $\begin{array}{l}\text { Floricultura, } \\
\text { Artesanía, } \\
\text { Servicios, } \\
\text { Construcción }\end{array}$ & $\begin{array}{l}\text { Agricultura, } \\
\text { Floricultura, } \\
\text { Artesanía, } \\
\text { Comercio, } \\
\text { otros Servicios }\end{array}$ & $\begin{array}{l}\text { Agricultura, } \\
\text { Artesanía, } \\
\text { Construcción, } \\
\text { servicios }\end{array}$ & $\begin{array}{l}\text { Agricultura, } \\
\text { Artesanía, } \\
\text { Construcción }\end{array}$ \\
\hline Agua & Entubada & Entubada y de pipa & De pozo & $\begin{array}{l}\text { Entubada y de } \\
\text { pipa }\end{array}$ \\
\hline Aguas negras & Hay drenaje & $\begin{array}{l}\text { No drenaje, } \\
\text { pero algunas casas } \\
\text { con fosas sépticas }\end{array}$ & No hay drenaje & No hay drenaje \\
\hline Bosque & $\begin{array}{l}\text { Vegetación } \\
\text { secundaria, } \\
\text { bosques de coníferas }\end{array}$ & $\begin{array}{l}\text { Vegetación } \\
\text { secundaria }\end{array}$ & $\begin{array}{l}\text { Vegetación } \\
\text { secundaria }\end{array}$ & $\begin{array}{l}\text { Algo de bosque } \\
\text { de pino encino }\end{array}$ \\
\hline Leña & Escasa & Muy escasa & Escasa & Disponible \\
\hline Contaminación por basura & $\begin{array}{l}\text { Fuerte, } \\
\text { pero hay servicio de } \\
\text { recolección }\end{array}$ & $\begin{array}{l}\text { Fuerte, } \\
\text { pero hay servicio de } \\
\text { recolección }\end{array}$ & $\begin{array}{l}\text { Fuerte, } \\
\text { no hay servicio de } \\
\text { recolección }\end{array}$ & $\begin{array}{l}\text { Moderado, } \\
\text { no hay servicio } \\
\text { de recolección }\end{array}$ \\
\hline
\end{tabular}

Fuente: INEGI, 2010 para los datos sobre número de habitantes y viviendas. Los demás datos provienen del trabajo de campo. 
Cuadro 2: Participantes en entrevistas por localidad, sexo y edad

\begin{tabular}{|c|c|c|c|c|}
\hline LOCALIDAD & $\begin{array}{l}\text { No. DE MUJERES } \\
\text { ENTREVISTADAS }\end{array}$ & EDADES & $\begin{array}{l}\text { No. DE HOMBRES } \\
\text { ENTREVISTADOS }\end{array}$ & EDADES \\
\hline Elambo & 3 & $24,47,64$ & 3 & $28,48,67$ \\
\hline Nachig & 1 & 55 & 1 & 43 \\
\hline Jech ch'entik & 1 & 33 & 1 & 48 \\
\hline Zinacantán & 4 & $19,40,44,74$ & 2 & 23,25 \\
\hline
\end{tabular}

Fuente: Elaboración propia de los autores.

\section{Cuadro 3: Participantes en talleres por localidad, descripción del grupo, sexo y grupos de edad}

\begin{tabular}{|c|c|c|c|c|c|}
\hline $\begin{array}{l}\text { LUGAR DONDE SE } \\
\text { LLEVÓ A CABO EL } \\
\text { TALLER }\end{array}$ & $\begin{array}{l}\text { DESCRIPCIÓN } \\
\text { DEL GRUPO }\end{array}$ & $\begin{array}{c}\text { No. DE } \\
\text { MUJERES } \\
\text { PARTICIPANTES }\end{array}$ & $\begin{array}{c}\text { No. DE } \\
\text { HOMBRES } \\
\text { PARTICIPANTES }\end{array}$ & EDAD & $\begin{array}{l}\text { No. } \\
\text { PARTICIPANTES }\end{array}$ \\
\hline Zinacantán & Estudiantes Prepa & 4 & 2 & $\begin{array}{c}\text { Jóvenes } \\
\text { (17-19 años) }\end{array}$ & 6 \\
\hline Zinacantán & Estudiantes Prepa & 3 & 9 & $\begin{array}{c}\text { Jóvenes } \\
\text { (16-19 años) }\end{array}$ & 12 \\
\hline Elambo & $\begin{array}{l}\text { Socias de MSV* y hom- } \\
\text { bres familiares }\end{array}$ & 15 & 3 & $\begin{array}{c}\text { Mixto } \\
\text { (15-60 años) }\end{array}$ & 18 \\
\hline Nachig & Socias de MSV* & 12 & & $\begin{array}{c}\text { Mixto } \\
\text { (20-50 años) }\end{array}$ & 12 \\
\hline Nachig & Socias de MSV* & 19 & & $\begin{array}{c}\text { Mixto } \\
\text { (18-70 años) }\end{array}$ & 19 \\
\hline San Cristóbal & Lideresas de MSV* & 11 & & $\begin{array}{c}\text { Mixto, } \\
\text { más jóvenes }\end{array}$ & 11 \\
\hline Zinacantán & Socias de MSV* & 7 & & $\begin{array}{c}\text { Mixto } \\
\text { (14-50 años) }\end{array}$ & 7 \\
\hline San Cristóbal & $\begin{array}{l}\text { Lideresas y socias de } \\
\text { MSV* }\end{array}$ & 14 & & $\begin{array}{c}\text { Mixto } \\
\text { (15-70 años) }\end{array}$ & 14 \\
\hline Jech ch'entik & Socias de MSV* & 18 & & $\begin{array}{c}\text { Mixto } \\
\text { (12-66 años) }\end{array}$ & 18 \\
\hline Total & & 103 & 14 & & 117 \\
\hline
\end{tabular}

*MSV significa "Mujeres Sembrando la Vida", grupo de mujeres que se organizan, entre otros temas, alrededor del microcrédito. Fuente: Elaboración propia de los autores. 


\section{Cuadro 4: Cambios ambientales principales identificados por los participantes en entrevistas y talleres en cuatro} comunidades de Zinacantán, Chiapas, 2009

\section{PROBLEMAS O CONFLICTOS}

Cambio productivo en la milpa; cambio de la milpa hacia la floricultura bajo invernadero. Consecuencias de floricultura: tala de árboles, escasez de agua, pérdida de autosuficiencia alimentaria, contaminación por agroquímicos.

Pérdida de fertilidad de suelos para milpa y problemas relacionados con agroquímicos: menos cosecha de maíz, milpa acostumbrada a fertilizantes, dependencia de agroquímicos caros.

Cambio climático: menos lluvia y menos seguido, frío y calor más intensos, más sequías.

Escasez y contaminación del agua en manantiales, arroyos y ríos. Conflictos por el recurso agua. Falta o escasez del agua entubada.

Deforestación, disminución y fragmentación de bosques. Consecuencias: menos agua, menos lluvia, menos plantas y hongos silvestres, menos animales silvestres, menos leña.

Más dependencia de alimentos comprados. Alimentación menos saludable. Pérdida de alimentos derivados de la milpa, disminución de animales domésticos.

Contaminación por basura: trae enfermedades, contaminación visual, contaminación de cuerpos de agua.

\section{VENTAJAS O CAMBIOS POSITIVOS}

Agua entubada o en pipa en algunas comunidades, ya no hay que ir a cargarla.

Servicio de recolección de basura en dos comunidades: menos basura regada.

Tiendas en las comunidades alejadas: disponibilidad de bienes de consumo.

Pavimentación de calles, construcción de carreteras, caminos: menos lodo, comunidades lejanas más accesibles, más cercanía relativa del pueblo (Zinacantán) y de San Cristóbal.

Casas de block y cemento percibidas como más cómodas y duraderas.

Fuente: Elaboración propia de los autores.

Cuadro 5: Cambios ambientales principales identificados en cuatro comunidades de Zinacantán, Chiapas, 2009

\begin{tabular}{||l||l||}
\hline \multicolumn{1}{||c||}{ COMUNIDAD } & \multicolumn{1}{c||}{ CAMBIOS AMBIENTALES PRINCIPALES IDENTIFICADOS: } \\
\hline \hline Zinacantán & Cambio hacia floricultura, deterioro del bosque, deterioro de la alimentación. \\
\hline \hline Nachig & Deterioro de la alimentación y escasez y conflictos por el agua. \\
\hline \hline Jech ch'entik & $\begin{array}{l}\text { Contaminación, escasez y conflictos por el agua, Contaminación por basura y deterioro del bosque } \\
\text { (especialmente falta de leña). }\end{array}$ \\
\hline \hline Elambo Bajo & Contaminación por basura y problemas con la milpa (especialmente pérdida de fertilidad). \\
\hline
\end{tabular}

Fuente: Elaboración propia de los autores. 